\title{
Determinantes del absentismo laboral según enfoque sociológico. Caso: empresa auxiliar automoción española
}

\author{
Ferro-Soto, Carlos* \\ García-Alonso, Ero** \\ Lareo-Lodeiro, Belén***
}

\section{Resumen}

El absentismo laboral es una de las conductas de abandono que más pérdidas ocasiona en casi todas las organizaciones del mundo. La identificación de sus causas permitirá incidir sobre el origen del problema, evitando sus consecuencias negativas. Así, este estudio persigue identificar los determinantes del comportamiento absentista desde un prisma sociológico. La investigación se ha basado en datos de una empresa auxiliar del sector del automóvil en España. Según la naturaleza de las variables, se ha recurrido a técnicas estadísticas paramétricas (ANOVA) y no paramétricas (test-t). El principal hallazgo ha sido identificar un foco absentista cuyo perfil responde a una persona de sexo femenino, no cualificada, encargada directamente de la fabricación de productos, realizando tareas estrictamente programadas sujetas a control de métodos y tiempos, que acostumbran a ser rutinarias y repetitivas, y dentro de un proceso de producción complejo. Se concluye que variables de corte sociológico como son el sexo, la categoría laboral y las condiciones estructurales del puesto de trabajo pueden ser factores determinantes del absentismo laboral.

Palabras clave: Absentismo laboral, condiciones de trabajo, enfoque sociológico, sector automoción.

\section{Recibido: 02-11-1.2 Aceptado: 25-02-14}

* Doctor en Ciencias Económicas y Empresariales por el departamento de Organización de Empresas y Marketing de la Universidad de Vigo (España). Profesor Titular de Universidad de Vigo, área Organización de Empresas. E-mail: cferro@uvigo.es

** Máster en Dirección y Gestión Laboral. Máster en Administración Integrada de Empresas. Doctorando en Programa Administración Integrada de Empresas (Universidad Vigo-España). Técnico de Recursos Humanos. E-mail: erogarciaalonso@gmail.com

*** MBA Escuela Negocios Caixanova (Vigo). Máster en Administración Integrada de Empresas. Doctoranda en Programa Administración Integrada de Empresas (Universidad Vigo-España). Abogada. Profesora Asociada Universidad de Vigo. E-mail: belen@estudionovecento.com 


\title{
Determinants for Absenteeism Seen from a Sociological Approach. Case: Spanish Automotive Supplier Company
}

\begin{abstract}
Absenteeism is one of the abandonment behaviors that occasions most losses in almost all organizations in the world. Identifying its causes will permit influencing the source of the problem, avoiding its negative consequences. This study aims to identify the determinants of absenteeism seen through a sociological prism. The research was based on data from a Spanish automotive supplier company. Depending on the nature of the variables involved, both parametric (ANOVA) and nonparametric (t-test) statistical techniques have been used. The main finding was to identify a focus of absentee workers whose profile describes a female person, who is not qualified, directly responsible for product manufacture and who performs strictly scheduled tasks subject to control of methods and times, which tend to be routine and repetitive within a complex production process. Conclusions are that sociological variables such as gender, employment status and structural conditions of the job may be factors that determine absenteeism.
\end{abstract}

Key words: Absenteeism, working conditions, sociological approach, automotive sector.

\section{Introducción}

Una de las conductas de abandono que mayor incidencia tiene y más pérdidas ocasionan en casi todas las organizaciones del mundo, es el absentismo laboral o inasistencia al trabajo del personal contratado (Ortiz y Samaniego, 1995). La tasa de absentismo laboral en Europa asciende al 4,6\%. Son Francia, Alemania y Bélgica los países más afectados, mientras que Italia y Austria arrojan las tasas más reducidas. En España, según datos manejados por la Confederación Española de Organizaciones Empresariales (CEOE en adelante), cada día un millón de personas no acude a su puesto de trabajo por enfermedades comunes y accidentes de trabajo, lo que supone una tasa media de ausencia al trabajo del 5,3\%. Estas bajas laborales tienen un costo anual de casi 13.000 millones de euros, lo que supone algo más de un $1 \%$ del PIB español. Si esta cantidad se desglosa por trabajador, el costo medio anual sería de unos 2.700 euros. Además, se estima que entre el $20 \%$ y el $30 \%$ de los trabajadores que no acuden a su puesto de trabajo lo hace de forma injustificada, lo que supone un costo de 8.000 millones de euros al año.

El absentismo laboral es un fenómeno universal que se erige como un grave problema que abarca una importante dimensión social, económica y humana, con repercusiones a tres niveles; a saber: empresarial, individual y social. Para la organización, el absentismo dificulta la planificación, resulta un obstáculo para la consecución de los objetivos o encarece el producto final. Para el trabajador, el absentismo genera tensiones entre los compañeros o puede disminuir sus percepciones económicas. Finalmente, para 
la sociedad, el absentismo, en algunos supuestos, supone una disminución de los ingresos a la Seguridad Social, el encarecimiento de productos o la deficiencia en los servicios.

En efecto, desde el punto de vista empresarial, el absentismo laboral impide a la compañía operar sus recursos con efectividad y eficiencia (Steers y Rhodes, 1978; Wright et al., 1995; Dalton y Mesch, 1991; Mason y Griffin, 2003; Randon et al., 2011; Battisti y Vallanti, 2013). Algunos de sus costos son fácilmente medibles, como lo son el costo de sustitución del personal ausente, el adiestramiento de los sustitutos y, posteriormente, de los empleados retornados, o las horas extraordinarias para compensar el trabajo no realizado. Hay que tener en cuenta que la simple sustitución de un empleado por otro no resuelve las consecuencias negativas que el absentismo ocasiona, debido a la familiaridad, conocimientos y práctica con que los empleados veteranos ejecutan su trabajo y de la que carecen los sustitutos (Moch y Fitzgibbons, 1985). A esto hay que añadir las quejas, protestas, agravios comparativos, accidentes laborales, disminución de productividad y calidad (Blumenfeld e Inman, 2009) que el absentismo recurrente genera. De otro lado, el absentismo también ocasiona ineficiencias en la planificación del dimensionamiento de la plantilla de la empresa, por cuanto que los efectivos necesarios para la realización del trabajo son calculados teniendo en cuenta las tasas de absentismo histórico.

A la par, el absentismo laboral constituye un fenómeno complejo (Rhodes y Steers (1990) consideran que la etiología del absentismo laboral está in- fluenciada por una constelación de diferentes factores interrelacionados, tanto individuales como organizacionales, y asociado a un número importante de consecuencias, tanto positivas como negativas. Este origen multifactorial del absentismo empuja a que se contemplen muchos aspectos en su análisis (Steers y Rhodes, 1984; Boada et al, 2004).

Así pues, debido tanto a su complejidad como a su importancia en términos de eficacia y eficiencia empresarial, en las últimas décadas se ha desarrollado un considerable cuerpo de investigación en torno al absentismo. Los aspectos críticos del absentismo recaen en encontrar la mejor forma para medirlo, en identificar sus principales causas y en descubrir qué hacer para reducir tal comportamiento (Rhodes y Steers, 1990). En este sentido, identificar los incentivos que mueven al trabajador a ausentarse del trabajo, permitirá incidir sobre tales estímulos, bien a nivel público-estatal como de empresa (Bayo, 2003; Bayo y Huerta 2000) y, a la postre, evitar las consecuencias negativas que de este fenómeno se derivan. La amplia y especial atención que se ha prestado al absentismo en la literatura científica para abordar estos aspectos críticos ha devenido en la formulación de tres modelos conceptuales principales de absentismo, derivados de las corrientes económicas, sicológicas y sociológicas.

Siguiendo la lógica económica, el absentismo se estudia como un fenómeno resultante de una elección entre trabajo y ocio (Barmby et al., 1991; Drago y Wooden, 1992; Frick y Malo, 2008). Bajo este prisma, los empleados estarán ausentes cuando los beneficios de no trabajar resulten ser mayores que los costos 
de no trabajar. Por tanto, se está considerando que el número de días que los empleados se ausentan de sus puestos de trabajo resultan de una decisión racional que tiene por objeto la maximización de su función de utilidad. Esta utilidad vendrá condicionada por los niveles deseados de ingresos, de ocio y de riesgo del trabajador.

Por su parte, el enfoque sicológico explica el comportamiento absentista basándose en la teoría de las expectativas de la motivación de los empleados desarrollada por Vroom (1964). Desde esta atalaya se asume que los empleados toman decisiones en base a la maximización de los resultados valorados (Steers y Rhodes, 1978). Se hace especial hincapié en las respuestas afectivas o emocionales de los empleados a condiciones organizacionales y del trabajo, y la evaluación consciente que hace el empleado para tomar la decisión de no asistir a su puesto de trabajo. Se supone que los empleados entran en las organizaciones con unas orientaciones preconcebidas, unos conocimientos y unas expectativas que, si no se cumplen, inducen al absentismo. Por tanto, los estudios realizados bajo esta perspectiva sicológica se centran en la motivación, la satisfacción y las actitudes hacia el puesto de trabajo. La motivación y la satisfacción laboral han sido los elementos que han tenido mayor importancia en el fenómeno de la sicosociologia social de todos los analizados por Chadwick-Jones et al en 1982. Entre los estudios llevados a cabo bajo el enfoque de la sicología aplicada, se han encontrado como predictores de conductas absentistas factores tales como los niveles de autoestima (Keller, 1983), la ansiedad y conciencia (Bernardin, 1977) o el clima laboral (Bourbonnais, 2005).

Finalmente, el enfoque sociológico para el estudio del absentismo incorpora conceptos que provienen de los dos modelos anteriores (económico y sicológico), a los que añade una dimensión orgánica relativa tanto a las características estructurales del puesto de trabajo como a circunstancias particulares del propio trabajador (Brooke, 1986; Brooke y Price, 1989). Así pues, bajo este enfoque, el absentismo ha sido estudiado como un fenómeno influido, de un lado, por factores internos de la organización del trabajo (concernientes al puesto de trabajo) tales como la centralización de la toma de decisiones, el trabajo en cadena, el tamaño de la unidad de trabajo o la repetitividad de la tarea. De otro lado, por factores particulares (concernientes al trabajador), tales como la edad, el sexo, el estado civil, la formación, la distancia del domicilio al lugar de trabajo o las responsabilidades externas derivadas de sus obligaciones familiares y personales fuera del trabajo (Løkke et al., 2007; Engström y Janson, 2009).

Bajo este enfoque, se hace posible desarrollar un perfil del trabajador absentista, definiendo variables sociológicas propias de las personas propensas al absentismo. Esto permitirá detectar los focos de absentismo dentro de la organización y diseñar estrategias para su seguimiento y control en aras de evitar las consecuencias negativas que de este fenómeno se derivan. De este modo se conseguiría la optimización de la gestión de los recursos humanos de la empresa, puesto que a menudo algunos factores que condicionan la falta de asistencia al 
trabajo se encuentran bajo el control de la administración de la compañía.

Así, el presente estudio tiene por objeto abordar el problema del absentismo laboral desde un enfoque sociológico. Bajo esta óptica, se persigue desarrollar un perfil del trabajador absentista, definiendo variables que hacen a las personas propensas al absentismo, como pueden ser el género, la edad, el nivel de ingresos por salario, la antigüedad en la empresa, el colectivo de trabajadores al que pertenece y la línea de producción en la que trabaja.

A tal fin, se han utilizado datos recogidos de una planta de producción dedicada a la transformación de plásticos. La planta forma parte de una división de una corporación extranjera dedicada a la automoción. Concretamente, la planta objeto de estudio se dedica exclusivamente a la producción de piezas para un fabricante de automóviles con filial de producción en Vigo (España). Pese a que la planta de producción desarrolla su actividad en el sector económico de la automoción como industria auxiliar, según la Clasificación Nacional de Actividades Económicas (en adelante CNAE) del 2009, la empresa se dedica a fabricación de productos de plástico, formando parte del sector químico.

De los datos disponibles de los 255 trabajadores de la planta, se han utilizado finalmente 243 , puesto que doce han resultado ser datos perdidos (outliers). Los datos referentes al absentismo fueron recogidos entre el 1 de enero de 2010 y el 31 de diciembre del mismo año a través del sistema de control de presencia de la planta de producción objeto de estudio. Dicho sistema lo forman las tarjetas de banda magnética individuales que cada trabajador posee, un reloj de fichar que registra las entradas y salidas del personal y el programa informático SCPWin. Es precisamente este software el que se utiliza para administrar los registros del reloj de fichar y extraer los datos referentes al absentismo empleados en el presente estudio. En dicho programa se tipifican las ausencias de los trabajadores como: visita a consultorio médico del trabajador, acompañamiento a visita a consultorio médico de un familiar del trabajador, hospitalización de un familiar del trabajador, defunción de un familiar del trabajador, horas sindicales e incapacidad transitoria. Los datos sociodemográficos se extrajeron de la base de datos del programa utilizado para confeccionar las nóminas de la empresa y se refieren al último día del año 2010.

Una vez comprendido el fenómeno absentista mediante una revisión exhaustiva de la literatura existente, desarrollada a lo largo de las secciones 2 y 3 , los datos han permitido explicar el origen de la propensión al absentismo debido a factores particulares (edad, nivel de ingresos por salario, antigüedad en la empresa) y características del puesto de trabajo (colectivo de trabajadores y línea de producción), dentro del tipo de actividad particular en el que centra su atención esta investigación. En este sentido, la contribución novedosa de este trabajo recae en la identificación de los determinantes clave del comportamiento absentista desde un prisma sociológico en el sector de actividad objeto de estudio. Para ello, se recurre a técnicas estadísticas paramétricas (ANOVA) y no paramétricas (test-t) en función de la naturaleza 
de las variables manejadas. Esto permite conocer los grupos con una mayor propensión absentista y, por ende, el principal hallazgo ha sido identificar el perfil de un foco absentista de trabajadores.

En la siguiente sección se estudian el concepto, significado y causas del absentismo con el objeto de desarrollar la línea argumental que lleva al planteamiento de las hipótesis. En las secciones 3 y 4 se presenta la metodología empírica y los resultados de la investigación en cuanto a los factores particulares y a las características del puesto de trabajo, respectivamente, como condicionantes del comportamiento absentista. Finalmente, en la sección 5 se procede a la discusión de los resultados y conclusiones.

\section{Absentismo laboral}

Diversos autores manifiestan que la definición del concepto de absentismo laboral supone un grave problema debido a que este fenómeno lleva unido una serie de comportamientos sin una relación aparente, y puede significar cosas diferentes según las personas y las situaciones en que se analice. Hasta ahora no existen teorías que abarquen todas las interpretaciones que se han elaborado sobre el tema. Por ello, cualquier estudio sobre absentismo laboral debe comenzar por clarificar el significado que se confiere al término.

El absentismo hace referencia a las conductas de ausencia del puesto de trabajo de los empleados durante el período de actividad laboral establecido por la organización (Chadwick-Jones et al., 1982; Ortiz y Samaniego, 1995). Estas ausen- cias al puesto de trabajo surgen por la imposibilidad de presentar varios estados mentales y funciones diversas con la actividad laboral de manera simultánea. No obstante, y dada la definición que manejamos en este trabajo, es posible diferenciar el ausentismo de otras formas de no asistencia organizadas con antelación (por ejemplo, días festivos y vacaciones anuales). A su vez, las ausencias no programadas pueden ser voluntarias, cuando se encuentran vinculadas con la motivación de un empleado para asistir, o involuntarias, cuando son debidas a la imposibilidad de asistir por motivos de enfermedad o lesión (Brooke y Price, 1989). Esta distinción lleva a que en algunos casos se utilice el vocablo ausentismo para referirse a la ausencia al trabajo como un acto involuntario y el término absentismo para describir acciones voluntarias en este terreno.

En efecto, el absentismo es un fenómeno que tiende a la opacidad y a la ocultación. Pese a que una de las principales causas por la que los empleados faltan regularmente al trabajo son los problemas de salud (Invalidez Laboral Transitoria-ILT-), en muchos casos se simula dolencias falsas legitimadas por facultativos autorizados para faltar al trabajo y, en ocasiones, lo hacen por largos períodos de tiempo, más allá del momento en que la dolencia, de existir realmente, haya remitido. Nova (1996) emplea el término absentismo medicalizado para referirse a este absentismo laboral simulado. Resulta muy difícil la comprobación y control de esta ILT con simulación puesto que se escudan en una relación médico-paciente, sustentada en la confianza, lealtad mutua y privacidad. 


\subsection{Absentismo laboral y circunstancias particulares del trabajador}

Estudios realizados a nivel individual han demostrado que las características personales de los trabajadores referidas al género, la edad, la situación familiar y la antigüedad tienen efectos sobre la frecuencia de absentismo (Kristensen et al., 2006). Cabe precisar que las características que conforman el género son construcciones socio-culturales puesto que son aprendidas y se pueden educar (Unger y Crawford, 1992; Velázquez, 2003). Además, tanto el género como la edad han sido utilizadas en todas las culturas como una manera de categorizar, influyendo ambos atributos en el modo de relacionarse las personas con su entorno (Deaux y Lewis, 1984; Lips, 1993; Matlin, 1993).

Diversos estudios han abordado trabajos de campo con el objeto de encontrar relaciones entre el absentismo laboral y el género. Así, recientemente, Markussen et al., (2011) hallaron que las tasas de absentismo femenino por enfermedad certificada eran superiores a las de los hombres, hasta en un $45-68 \%$ (tasa absentismo hombre-mujer\%) para enfermedades leves y en un $26-43 \%$ (tasa absentismo hombre-mujer\%) para enfermedades graves. Sin embargo, encontraron que las diferencias de género en las tasas de recuperación eran mucho más pequeñas, tendiendo las mujeres a recuperarse un poco más lentamente que los hombres de enfermedades graves, sobre todo si tienen hijos.

Asimismo, Ichino y Moretti (2009) afirman que en la mayoría de los países, las mujeres son más absentistas que los hombres. Para buscar la explicación de estas desigualdades se fijaron en las diferencias biológicas de género, llevando a cabo un estudio entre empleadas bancarias italianas. De este modo, encontraron que la menstruación puede contribuir a las diferencias de género en el absentismo, basándose en la evidencia de que las ausencias de las mujeres por debajo de la edad de 45 años siguen ciclos de 28 días, mientras que las ausencias de los hombres y de mujeres mayores de 45 años de edad no lo hacen. Contrariamente, Rockoff y Herrmann (2012) analizaron el absentismo en el colectivo de los docentes y no encontraron ninguna evidencia de que el absentismo femenino aumente en un ciclo de 28 días. Además, estos autores demuestran falta de robustez en la evidencia de los ciclos de 28 días hallada por Ichino y Moretti (2009) debido a los datos utilizados.

Con la incorporación de la mujer al mercado laboral, desarrollando trabajos retribuidos tanto por cuenta ajena como propia, en las sociedades de los países industrializados desde finales de la II Guerra Mundial, se ha impulsado el importante incremento de familias en que ambos cónyuges trabajan (López-Cordón, 1998; Greenhaus y Powell, 2006). Ante los cambios en la distribución del tiempo doméstico que genera este nuevo escenario familiar, Zick y McCullough (1991) contemplan dos perspectivas: la optimista, que prevé una menor dedicación de la mujer en las tareas domésticas y cierto cambio en los roles sexuales, y la pesimista, que pronostica un aumento y sobrecarga de tareas para la mujer, de- 
nominado por Hochschilld (1989) como segundo turno o doble jornada.

En esta línea argumental, Behrend (1959), con respecto a la función social asignada a cada sexo y a la categoría ocupacional, comprueba que las ausencias se manifiestan de forma diferente entre los hombres y las mujeres, y los elementos que marcan la diferenciación son el tipo de trabajo y las responsabilidades familiares. Por su parte, Isambert-Jamati (1960) investiga la fuerza que tienen los factores familiares y profesionales, y la adaptación y el nivel de cualificación de las trabajadoras. Su conclusión fue que no se puede explicar el absentismo femenino por la incidencia de los determinantes biológicos o familiares, ya que las mujeres casadas faltan más que las mujeres solteras. Asimismo, Paringer (1983) observa las tasas de ausencia en ambos sexos y obtiene que las causas son la desigualdad de sueldos y la carencia de flexibilidad de horario, aunque las empleadas restringen más sus faltas por enfermedad que los hombres y predominan más las motivadas por factores familiares.

Asumiendo la perspectiva pesimista de Zick y McCullough (1991) como la más realista, se puede esperar que las ausencias femeninas son superiores a las masculinas por el doble de tareas que deben desempeñar las trabajadoras, al tener que compaginar los horarios de trabajo con el de la guardería de los niños, pedir permisos extra para ir al médico con ellos, entre otras responsabilidades y tareas familiares. Además, considerando los posibles determinantes biológicos que pueden ocasionar un efecto promotor del absentismo femenino, se puede plantear la siguiente hipótesis:
H1: Existen diferencias significativas en el comportamiento absentista atendiendo al género de los trabajadores

Markussen et al., (2011) encuentran que la probabilidad de ausencia por enfermedad se reduce significativamente a medida que aumenta la edad del trabajador hasta los 45 años. De hecho, según su estudio, en igualdad de condiciones la tasa de ausencia por enfermedad leve es un $70 \%$ más alto en un empleado de 30 años que en uno de 45 años. Sin embargo, la tasa se reduce a un $10 \%$ cuando se habla de ausencia por enfermedades graves. Por encima de 45 años, las tasas tienden a estabilizarse para enfermedades leves o a subir para enfermedades graves. Por su parte, la probabilidad de recuperación a partir de una enfermedad disminuye uniformemente con la edad.

Dado que, por lo general, las condiciones de salud de los individuos se deterioran con la edad, las tasas de absentismo deberían ir aumentando a medida que aumenta la edad del empleado. Esto se amplifica, si se atiende a que son las enfermedades graves las que requieren un mayor número de días para su recuperación. Por tanto, en estos términos, se plantea la siguiente hipótesis:

$H 2$. Existe una relación significativa y positiva entre días de ausencia por motivos de ILT y la edad del trabajador

En la medida en que el absentismo se manifiesta como una respuesta del trabajador frente a condiciones laborales que le resultan insatisfactorias, el comportamiento absentista debería reducirse con la antigüedad en la empresa, entendiendo que los trabajadores más antiguos han tenido más tiempo para encontrar un trabajo más acorde con sus de- 
seos y, por ende, más satisfactorio (Martocchio, 1989). Por tanto, no tendrá una menor propensión a enmascarar 'huidas' de su puesto de trabajo en forma de absentismo medicalizado en términos de Nova (1996). En ese sentido, se plantea la siguiente hipótesis:

H3. Existe una relación significativa e inversa entre días de ausencia por motivos de ILT y la antigüedad del empleado

Asimismo, para el empleado, el absentismo puede ser una forma de huida motivada por la baja remuneración económica. De hecho, algunos autores (Allen, 1981; Dunn y Youngblood, 1986) han demostrado que la reducción de las tasas de ausencia se asocia, entre otros factores, a un mayor salario. Más recientemente, Markussen et al., (2011) llegan a las mismas conclusiones, confirmando que de forma general, el absentismo se reduce a medida que aumenta la retribución salarial, en relación con el número de horas trabajadas. Más concretamente, hallaron que, manteniendo los demás factores constantes (condiciones ceteris paribus), dentro del grupo de trabajadores a tiempo completo, los miembros que se encontraban en el cuartil superior de ganancias presentaban unos ratio de absentismo un $42 \%$ más bajo que aquellos que se encontraban dentro del primer cuartil de retribución salarial. Ante tales evidencias previas, se considera la siguiente hipótesis:

H4: Existe relación significativa y en sentido inverso entre el nivel retributivo y el número de horas de ausencia.

\subsection{Absentismo laboral y características estructurales del puesto de trabajo}

La motivación de un trabajador para asistir a su puesto de trabajo se verá afectado por la forma en la que está diseñado y dirigido el trabajo. Los trabajos rutinarios, con un fuerte grado de repetición (Price y Mueller, 1981), y poco gratificante, junto con la falta de oportunidades de participación en la toma de decisiones sobre aspectos que afectan directamente al trabajo desempeñado (White y Ruh, 1973) están asociados con mayores niveles de absentismo (Hackman y Lawler, 1971; Price y Mueller, 1981, 1986; Curry et al., 1985; Brooke, 1986; Tetrick y LaRocco, 1987; Arsenault y Dolan, 1983; Brooke y Price, 1989; Iverson et al, 1995). En consecuencia, los obreros (trabajadores de cuello azul o blue-collar workers) presentan unos niveles de absentismo superiores que los empleados de cuello blanco por cuanto que el trabajo de estos últimos es menos rutinario, más gratificante y más participativo (Price, 1972; Chadwick-Jones et al., 1982;. Edwards y Scullion, 1982; Arsenault y Dolan, 1983). En función de ello se plantea la siguiente hipótesis:

H5: Existen diferencias significativas en el comportamiento absentista si se atiende a la categoría laboral de los trabajadores.

Del mismo modo, a igualdad de categoría laboral, si las condiciones estructurales del puesto de trabajo son diferentes, cabría encontrar diferentes compor- 
tamientos absentistas. Por tanto, bajo el supuesto de que distintas líneas de producción suponen distintas características de trabajo desarrollado por los empleados, entonces se puede plantear la siguiente hipótesis:

H6: Existen diferencias significativas en el comportamiento absentista si se atiende a la línea de producción en la que desarrollan su actividad los trabajadores.

\subsection{Modelo para la caracterización del perfil absentista del trabajador}

El modelo teórico-explicativo debe ser correlativo a la medición del fenómeno a estudiar. A tal fin, en este trabajo se ha utilizado como variable explicada el absentismo, medida a través del número de días que el trabajador se ausenta de su puesto de trabajo por ILT. Por término medio, los trabajadores de la empresa objeto de estudio se ausentan por este motivo 78.2587 días/año, con una desviación típica de 246.35341 días/año. Como variables explicativas se han considerado dos grupos: variables referidas a condiciones particulares del trabajador, tales como sexo, edad, antigüedad en la empresa y retribución, y las variables referidas a las condiciones estructurales del puesto de trabajo, que vienen determinadas por la categoría laboral y la línea de producción en la que desarrolla su actividad el trabajador (Gráfico 1).

\section{Perfil absentista del trabajador según características personales del trabajador}

Para contrastar la primera hipótesis planteada y conocer si existen diferencias significativas en el comportamiento absentista atendiendo a la variable género, se realizó un test ANOVA de un factor.

\section{Gráfico 1}

Modelo para la caracterización del perfil absentista del trabajador

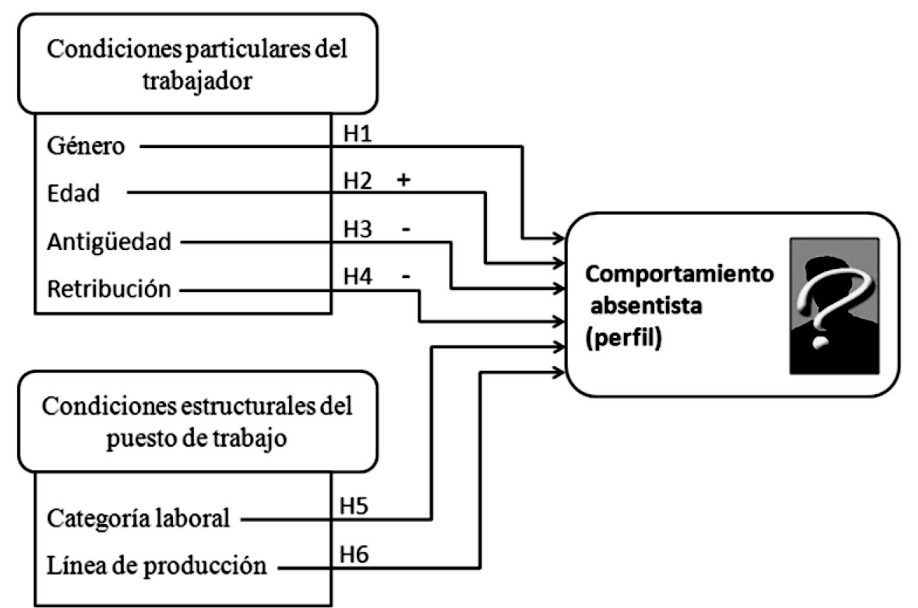

Fuente: Elaboración propia. 
Como se colige de la información recogida en la Tabla 1, el test arrojó diferencias significativas entre los niveles de esta variable $(F=4.748 ; p=.030)$, lo que permite apoyar esta hipótesis.

Por tanto, las diferencias entre las medias de cada grupo mostradas en la Tabla 2 son significativas. Se observa como, por término medio, las mujeres son más absentistas (con una media de 142,96 días/año) que los hombres (con una media de 60,21 días/año) cuando la causa de la ausencia atiende a motivos de ILT. Sin embargo, cabe resaltar que la desviación típica respecto a la media es aproximadamente 125 días superior para mujeres que para hombres.

Sin embargo, cuando se realiza la ANOVA de un factor (Tabla 3) para días de ausencia justificados por razones diferentes a la ILT (visita a consultorio médico del trabajador, acompañamiento a visita a consultorio médico de un familiar del trabajador, hospitalización de un familiar del trabajador, defunción de un familiar del trabajador y horas sindicales) las diferencias de medias atendiendo a los dos niveles de género no resultan significativas $(F=2.149 ; p=.144)$.

Las demás características personales del trabajador consideradas son la antigüedad del individuo (años), el salario bruto teórico anual y la edad del individuo $y$, en base a ellas se han planteado las hipótesis segunda, tercera y cuarta. Las medias y desviaciones típicas correspondientes a estas variables se muestran en la Tabla 4.

\section{Tabla 1}

ANOVA de un factor para IT y género

\begin{tabular}{cccccc}
\hline & Suma de cuadrados & gl & Media cuadrática & F & Sig. \\
\hline Inter-grupos & 283765,667 & 1 & 283765,667 & 4,748 &, 030 \\
Intra-grupos & 14403214,713 & 241 & 59764,376 & & \\
Total & 14686980,380 & 242 & & & \\
\hline
\end{tabular}

Fuente: Elaboración propia.

\section{Tabla 2}

\section{Descriptivos del proceso de incapacidad transitoria del individuo según género}

\begin{tabular}{|c|c|c|c|c|c|c|c|c|}
\hline & \multirow[b]{2}{*}{$\mathrm{N}$} & \multirow[b]{2}{*}{ Media } & \multirow[b]{2}{*}{$\begin{array}{c}\text { Desviación } \\
\text { típica }\end{array}$} & \multirow[b]{2}{*}{$\begin{array}{l}\text { Error } \\
\text { típico }\end{array}$} & \multicolumn{2}{|c|}{$\begin{array}{c}\text { Intervalo de } \\
\text { confianza para la } \\
\text { media al } 95 \%\end{array}$} & \multirow[t]{2}{*}{ Mínimo } & \multirow[t]{2}{*}{ Máximo } \\
\hline & & & & & $\begin{array}{l}\text { Límite } \\
\text { inferior }\end{array}$ & $\begin{array}{l}\text { Límite } \\
\text { superior }\end{array}$ & & \\
\hline Hombre & 190 & 60,2103 & 212,15378 & 15,39125 & 29,8496 & 90,5710 &, 00 & 1503,62 \\
\hline Mujer & 53 & 142,9604 & 336,73976 & 46,25476 & 50,1434 & 235,7774 & ,00 & 1584,00 \\
\hline Total & 243 & 78,2587 & 246,35341 & 15,80358 & 47,1286 & 109,3888 &, 00 & 1584,00 \\
\hline
\end{tabular}

Fuente: Elaboración propia. 


\section{Tabla 3}

\section{ANOVA de un factor para ausencias no IT y género}

\begin{tabular}{||c|c|c|c|c|c||}
\hline & Suma de cuadrados & gl & Media cuadrática & F & Sig. \\
\hline Inter-grupos & 255,420 & 1 & 255,420 & 2,149 &, 144 \\
\hline Intra-grupos & 28650,759 & 241 & 118,883 & & \\
\hline Total & 28906,179 & 242 & & & \\
\hline
\end{tabular}

Fuente: Elaboración propia.

\section{Tabla 4}

\section{Estadísticos descriptivos correspondientes a las variables antigüedad, salario bruto teórico anual y edad}

\begin{tabular}{cccc}
\hline & Media & Desviación típica & N \\
\hline Antigüedad del individuo (años) & 11,0274 & 7,94289 & 243 \\
Salario bruto teórico anual & 23823,8965 & 9090,40625 & 243 \\
Edad del individuo & 37,51 & 7,118 & 243 \\
\hline
\end{tabular}

Fuente: Elaboración propia.

Sin embargo, dado que todas estas variables tienen un componente temporal, se puede desconfiar de la existencia de correlaciones fuertes entre ellas, lo que puede desvirtuar los resultados de los contrastes que se realicen para la confirmación de las hipótesis relacionadas. Por ello, se procede a su previa comprobación mediante el coeficiente de correlación de Pearson en la matriz de co- rrelaciones mostrada en la Tabla 5 . Se desprende que las variables edad y antigüedad se encuentran fuertemente correlacionadas ( $r=.827$ ). Por el contrario, la correlación entre antigüedad y salario es más débil ( $r=.371)$, aunque también significativa (significación unilateral =,000).

Los datos anteriores arrojados por la matriz de correlaciones conducen a desechar dos de estas tres variables y a uti-

Tabla 5

Correlaciones entre las variables Antigüedad, Salario y Edad

\begin{tabular}{ccc}
\hline Variables & Correlación de Pearson & Sig. (unilateral) \\
\hline $\begin{array}{c}\text { Antigüedad del individuo en años * Salario } \\
\text { bruto teórico anual }\end{array}$ &, 371 &, 000 \\
$\begin{array}{c}\text { Antigüedad del individuo en años * Edad } \\
\text { del individuo }\end{array}$ &, 827 &, 000 \\
$\begin{array}{c}\text { Edad del individuo * Salario bruto teórico } \\
\text { anual }\end{array}$ &, 411 &, 000 \\
\hline
\end{tabular}

Fuente: Elaboración propia. 
lizar sólo una en el análisis. No obstante, mediante el coeficiente de correlación de Pearson en la matriz de correlaciones mostrada en la Tabla 6 entre la variable explicada, comportamiento absentista medida en días de ausencia motivados por proceso de ILT, y las variables antigüedad, salario y edad, se muestra que tal correlación no es significativa. El valor de los coeficientes indica que existe una relación muy débil entre la variable dependiente y las variables explicativas Antigüedad $(r=-, 059)$ y Salario $(r=-, 083)$. Esta relación es nula para la variable Edad $(r=-, 000)$. Quizás este último caso se puede justificar por el hecho de que existe poca variabilidad en los valores tomados por esta variable, puesto que si se presta atención a los descriptivos mostrados en la Tabla 4, por término medio la edad de los trabajadores de la empresa base de este estudio ronda los 37 años, con una desviación típica en torno a 7 años. Esto quiere decir que la edad de la plantilla ronda entre los 30 y 44 años, aproximadamente.

A la vista de estos resultados, se tiene que rechazar las hipótesis segunda, tercera y cuarta, al no tener las variables Antigüedad, Salario y Edad capacidad explicativa sobre la variabilidad experi- mentada por el absentismo. Consecuentemente, el contraste conduce a afirmar que no existe relación significativa entre la antigüedad, el nivel retributivo y la edad con el número de horas de ausencia por motivos de ILT. Pese a la falta de significatividad de las correlaciones y la debilidad de las mismas, sí se puede observar que de existir algún tipo de relación entre absentismo con Antigüedad y Salario, esta sería en sentido inverso, tal y como se desprende de los signos (negativos) de los correspondientes coeficientes de correlación, y tal y como se había predicho en las hipótesis tercera y cuarta.

\section{Personalidad absentista del trabajador según características estructurales del puesto de trabajo}

La quinta de las hipótesis planteadas hace referencia a la variable categoría laboral, que se mide a través de tres niveles; a saber: direct labour (DL), indirect hourly $(\mathrm{IH})$ y salary (S). Mientras que en terminología anglosajona el personal de estructura (personal de "oficina" o salary) se identifica con los white-collar workers, los trabajadores de taller direct labour (mano de obra directa-MOD-) y los

Tabla 6

Correlación de Proceso de ILT con Antigüedad, Salario y Edad

\begin{tabular}{ccc}
\hline Correlación de Proceso de ILT con & Correlación de Pearson & Sig. (unilateral) \\
\hline Antigüedad del individuo en años &,- 059 &, 180 \\
Salario bruto teórico anual &,- 083 &, 099 \\
Edad del individuo &, 000 &, 497 \\
\hline
\end{tabular}

Fuente: Elaboración propia. 
indirect hourly (mano de obra indirecta -MOI-) se corresponden con los blue-collar workers.

El personal de estructura lleva a cabo trabajos para los cuales es necesaria una alta cualificación y desarrollan su trabajo a jornada partida. Por su parte, el personal de taller suele ser de baja y media cualificación y se encuentra repartido en turnos de trabajo, con jornada continuada. En esta categoría, los indirect hourly no se encargan directamente de la fabricación de productos (tarea propia de los direct labour) sino que ocupan puestos de apoyo como mantenimiento, carretilleros de logística, limpiadores de maquinaria, control de calidad y jefes de equipo. A diferencia de los direct labour, su trabajo no está tan estrictamente programado, sus tareas no acostumbran a ser tan rutinarias y repetitivas, requieren de una mayor cualificación y no resulta habitual la realización de un control de métodos y tiempos de sus tareas.

A nivel retributivo, el salario de los whitecollars depende del grado de responsabilidad desempeñado, encontrándose por encima del mínimo establecido para su grupo profesional por convenio colectivo, normalmente definido por pactos individuales formalizados a través del contrato de trabajo. Asimismo, pueden disfrutar de una parte variable en función de objetivos, pero raras veces dependiendo del número de horas de presencia. Además, se pueden establecer diferentes escalas de promoción salarial por periodos de tiempo que no impliquen movilidad funcional. Por su parte, los bluecollars acostumbran a ser retribuidos por horas de trabajo y su retribución viene definida en convenio colectivo y únicamente disfrutan de promociones salariales con carácter colectivo a través de pactos entre Comité de Empresa y Dirección, o variaciones en la escala retributiva reconocidas por convenio colectivo.

Si se atiende a estos tres tipos de categorías laborales (direct labour, indirect hourly y salary), el ANOVA de un factor realizado (Tabla 7) arrojó diferencias significativas entre los niveles de esta variable $(F=3.352 ; p=.037)$.

Posteriormente se procedió a efectuar las comparaciones dos a dos, recurriendo a estadísticos ANOVA no asumiendo varianzas iguales, guiados por los resultados de la prueba de homogeneidad de varianzas de Levene (Tabla 8).

El estadístico utilizado para realizar las comparaciones dos a dos ha sido el de Games-Howell, por ser, en términos generales, el que mejor controla la tasa de error en diferentes situaciones. Así, se

\section{Tabla 7}

ANOVA de un factor para IT y categoría laboral

\begin{tabular}{cccccc}
\hline & Suma de cuadrados & gl & Media cuadrática & F & Sig. \\
\hline Inter-grupos & 399161,161 & 2 & 199580,581 & 3,352 &, 037 \\
Intra-grupos & 14287819,219 & 240 & 59532,580 & & \\
Total & 14686980,380 & 242 & & & \\
\hline
\end{tabular}

Fuente: Elaboración propia. 


\section{Tabla 8 \\ Prueba de homogeneidad de \\ varianzas}

\begin{tabular}{cccc}
\hline $\begin{array}{c}\text { Estadístico de } \\
\text { Levene }\end{array}$ & gl1 & gl2 & Sig. \\
\hline 9,397 & 2 & 240 &, 000 \\
\hline
\end{tabular}

Fuente: Elaboración propia.

ha podido observar que tan sólo existían diferencias significativas $(p=.008)$ entre el grupo direct labour y el indirect hourly (Tabla 9), lo que ha permitido confirmar parcialmente la quinta hipótesis.

Se puede observar en la Tabla 10 como la media de días de ausencia por ILT es 91 días/año superior para los direct labour (media $=111.5979)$ frente a los indirect hourly (media $=20.3871$ ).

Para completar los resultado obtenidos sobre absentismos por ILT en base a la categoría laboral, se puede decir que estas diferencias significativas de medias entre los distintos niveles de esta variable no se confirman si se atiende a ausencias motivadas por razones diferentes a ILT (motivos familiares, visita a consultorio médico del trabajador $u$ horas sindicales) como se puede observar en la Tabla 11 $(F=1.139 ; p=.322)$.

La última de las hipótesis planteadas (hipótesis seis) está relacionada con las diferentes condiciones estructurales del puesto de trabajo que puedan existir entre líneas de producción diferentes. A este respecto, la producción de la planta objeto de este estudio está estructurada en dos líneas y, por ello, la variable línea de producción toma dos valores: línea A y línea B.

Cada una de estas líneas se dedica a una familia diferente de productos. Ambos productos son complejos y requieren un alto grado de calidad ya que son partes estéticas del vehículo. En ambas líneas existen tres procesos de fabricación comunes (inyección, pintura y montaje). Sin embargo, la línea A cuenta a mayores con dos procesos particulares, la termo-

\section{Tabla 9}

\section{Comparaciones múltiples para la variable categoría laboral con IT} Games-Howell

(I) Taller

(J) Taller medias (I-J)
Diferencia de Error típico
Sig. Intervalo de confianza al $95 \%$

\begin{tabular}{cccc} 
& & \multicolumn{2}{c}{$95 \%$} \\
\cline { 3 - 4 } & & $\begin{array}{c}\text { Límite } \\
\text { inferior }\end{array}$ & $\begin{array}{c}\text { Límite } \\
\text { superior }\end{array}$ \\
\hline 9,97526 &, 008 & 20,4054 & 162,0162 \\
45,68754 &, 361 & $-46,8154$ & 172,2696 \\
29,97526 &, 008 & $-162,0162$ & $-20,4054$ \\
3,92416 &, 794 & $-134,2228$ & $-77,2555$ \\
45,68754 &, 361 & $-172,2696$ & 46,8154 \\
3,92416 &, 794 & 77,2555 & 134,2228 \\
\hline
\end{tabular}

* La diferencia de medias es significativa al nivel 0.05 .

Fuente: Elaboración propia. 


\section{Tabla 10}

\section{Descriptivos del proceso de incapacidad transitoria del individuo según categoría laboral}

\begin{tabular}{|c|c|c|c|c|c|c|c|c|}
\hline & \multirow[b]{2}{*}{$\mathrm{N}$} & \multirow[b]{2}{*}{ Media } & \multirow[b]{2}{*}{$\begin{array}{c}\text { Desviación } \\
\text { típica }\end{array}$} & \multirow[b]{2}{*}{$\begin{array}{l}\text { Error } \\
\text { típico }\end{array}$} & \multicolumn{2}{|c|}{$\begin{array}{c}\text { Intervalo de } \\
\text { confianza para la } \\
\text { media al } 95 \%\end{array}$} & \multirow[b]{2}{*}{ Mínimo } & \multirow[b]{2}{*}{ Máximo } \\
\hline & & & & & $\begin{array}{l}\text { Límite } \\
\text { inferior }\end{array}$ & $\begin{array}{l}\text { Límite } \\
\text { superior }\end{array}$ & & \\
\hline Direct labour & 142 & 111,5979 & 273,88690 & 22,98408 & 66,1599 & 157,0358 & ,00 & 1584,00 \\
\hline Indirect hourly & 62 & 20,3871 & 151,51036 & 19,24183 & $-18,0893$ & 58,8635 & ,00 & 1192,00 \\
\hline Salary & 39 & 48,8708 & 246,58519 & 39,48523 & $-31,0629$ & 128,8044 & ,00 & 1503,62 \\
\hline Total & 243 & 78,2587 & 246,35341 & 15,80358 & 47,1286 & 109,3888 &, 00 & 1584,00 \\
\hline
\end{tabular}

Fuente: Elaboración propia.

Tabla 11

ANOVA de un factor para ausencias no IT y categoría laboral

\begin{tabular}{cccccc}
\hline & Suma de cuadrados & gl & Media cuadrática & F & Sig. \\
\hline Inter-grupos & 271,802 & 2 & 135,901 & 1,139 &, 322 \\
Intra-grupos & 28634,377 & 240 & 119,310 & & \\
Total & 28906,179 & 242 & & & \\
\hline
\end{tabular}

Fuente: Elaboración propia.

compresión y el termoforrado, que hacen que su proceso de producción resulte más complejo. Esta línea cuenta con un menor número de efectivos ( 55 frente a los 96 de la línea B) ya que, a pesar de ser el proceso más complejo que el de la línea $B$, sus productos son menos voluminosos y, por ende, requieren un menor número de personas para la manipulación de las piezas. En ambas líneas el perfil profesional de la mano de obra directa es muy similar, no habiendo diferencias que afecten a los procesos de selección de personal en función de la línea de producción. La media de días de ausencia por ILT en la línea A (163.0840 días/año) es superior que en la línea B (63.5550 días/año). Los valores de los descriptivos se muestran en la Tabla 12.

Para comprobar que estas medias son significativas, se procede a realizar un test ANOVA de un factor en la variable explicada (ILT) y la línea de producción. El resultado de este test (Tabla 13) indica que dichas diferencias de medias sí resultan ser significativas entre los niveles de esta variable $(F=5.144 ; p=.025)$, por cuanto que se puede confirmar la presunción planteada en la sexta hipótesis.

Adicionalmente, estas diferencias resultan ser también significativas para los niveles tomados por esta variable 


\section{Tabla 12}

\section{Descriptivos del proceso de incapacidad transitoria del individuo según línea de producción}

\begin{tabular}{|c|c|c|c|c|c|c|c|c|}
\hline & \multirow[b]{2}{*}{$\mathrm{N}$} & \multirow[b]{2}{*}{ Media } & \multirow[b]{2}{*}{$\begin{array}{l}\text { Desviación } \\
\text { típica }\end{array}$} & \multirow[b]{2}{*}{$\begin{array}{l}\text { Error } \\
\text { típico }\end{array}$} & \multicolumn{2}{|c|}{$\begin{array}{l}\text { Intervalo de confianza } \\
\text { para la media al } 95 \%\end{array}$} & \multirow[b]{2}{*}{ Mínimo } & \multirow[b]{2}{*}{ Máximo } \\
\hline & & & & & $\begin{array}{l}\text { Límite } \\
\text { inferior }\end{array}$ & $\begin{array}{l}\text { Límite } \\
\text { superior }\end{array}$ & & \\
\hline$A$ & 55 & 163,0840 & 345,04846 & 46,52632 & 69,8043 & 256,3637 &, 00 & 1584,00 \\
\hline B & 96 & 63,5550 & 194,75586 & 19,87719 & 24,0938 & 103,0162 &, 00 & 1424,00 \\
\hline Total & 151 & 99,8073 & 263,04466 & 21,40627 & 57,5105 & 142,1040 &, 00 & 1584,00 \\
\hline
\end{tabular}

Fuente: Elaboración propia.

Tabla 13

ANOVA de un factor para IT y línea de producción

\begin{tabular}{cccccc}
\hline & Suma de cuadrados & gl & Media cuadrática & F & Sig. \\
\hline Inter-grupos & 346382,750 & 1 & 346382,750 & 5,144 &, 025 \\
Intra-grupos & 10032490,907 & 149 & 67332,154 & & \\
Total & 10378873,658 & 150 & & & \\
\hline
\end{tabular}

Fuente: Elaboración propia.

cuando las ausencias vienen justificadas por motivos familiares, visita a consultorio médico del trabajador u horas sindicales. Esta afirmación se realiza a la vista de los resultados $(F=6.898 ; p=.010)$ arrojados por el test ANOVA para un factor realizado (Tabla14).
En la Tabla 15 se resumen todos los contrastes realizados, informando sobre las variables manejadas en cada hipótesis, la técnica de contraste utilizada y el resultado del contraste. Esta información resulta de utilidad para el planteamiento de las conclusiones de este trabajo.

\section{Tabla 14}

ANOVA de un factor para ausencias no IT y línea de producción

\begin{tabular}{|c|c|c|c|c|c||}
\hline & Suma de cuadrados & gl & Media cuadrática & F & Sig. \\
\hline Inter-grupos & 571,175 & 1 & 571,175 & 6,898 &, 010 \\
\hline Intra-grupos & 12336,957 & 149 & 82,798 & & \\
\hline Total & 12908,132 & 150 & & & \\
\hline
\end{tabular}

Fuente: Elaboración propia. 


\section{Tabla 15}

Resumen de los contrastes de hipótesis realizados

\begin{tabular}{|c|c|c|c|}
\hline $\mathrm{N}^{\circ}$ hip. & $\begin{array}{c}\text { Variables relacionadas } \\
\text { en la hipótesis }\end{array}$ & Técnica de contraste & Resultado del contraste \\
\hline Hip. 1 & Género y Ausencia por ILT & Prueba ANOVA de un factor & Aceptada \\
\hline Hip. 2 & $\begin{array}{l}\text { Edad y Ausencia por ILT } \\
\quad \text { (relación positiva) }\end{array}$ & $\begin{array}{c}\text { Coeficiente de correlación de } \\
\text { Pearson }\end{array}$ & Rechazada \\
\hline Hip. 3 & $\begin{array}{l}\text { Antigüedad y Ausencia por ILT } \\
\text { (relación inversa) }\end{array}$ & $\begin{array}{c}\text { Coeficiente de correlación de } \\
\text { Pearson }\end{array}$ & Rechazada \\
\hline Hip. 4 & $\begin{array}{l}\text { Nivel retributivo y Ausencia por } \\
\text { ILT (relación inversa) }\end{array}$ & $\begin{array}{c}\text { Coeficiente de correlación de } \\
\text { Pearson }\end{array}$ & Rechazada \\
\hline Hip. 5. & $\begin{array}{c}\text { Categoría laboral y Ausencia } \\
\text { por ILT }\end{array}$ & Prueba ANOVA de un factor & Aceptada parcialmente* \\
\hline Hip. 6 & $\begin{array}{l}\text { Línea de producción y } \\
\text { Ausencia por ILT }\end{array}$ & Prueba ANOVA de un factor & Aceptada \\
\hline
\end{tabular}

*sólo para Direct labour e Indirect hourly

Fuente: Elaboración propia.

\section{Conclusiones}

El absentismo laboral o inasistencia al trabajo del personal contratado es un fenómeno universal del que devienen importantes efectos negativos no tan solo a nivel individual y social, sino también a nivel económico, ocasionando importantes pérdidas empresariales al impedir que las compañías exploten sus recursos de forma efectiva y eficiente, e imprimiendo, además, graves desequilibrios en las arcas de la Seguridad Social de los estados.

Debido su carácter complejo resultante de su origen multifactorial y dada su importancia sobre la productividad empresarial, en las últimas décadas se han multiplicado las investigaciones en torno al absentismo con el objeto de diseñar estrategias que permitan su seguimiento y control, para que, a la postre, se pueda optimizar la gestión de los recursos humanos de la empresa. Estos estudios se han desarrollado desde un enfoque económico, un enfoque sicológico y un enfoque sociológico.

El enfoque sociológico incorpora conceptos de los otros dos modelos y añade una dimensión orgánica relativa a, de un lado, las condiciones estructurales del puesto de trabajo, como pueden ser la centralización de la toma de decisiones, el trabajo en cadena, el tamaño de la unidad de trabajo o la repetitividad de la tarea, $y$, de otro lado, las circunstancias particulares del trabajador, tales como edad, sexo, estado civil, formación, o factores externos a los trabajadores como la distancia del domicilio al lugar de trabajo o las responsabilidades familiares y personales.

Enmarcar el estudio del comportamiento absentista en las arenas del enfoque sociológico hace posible determinar un perfil del trabajador absentista, que permitirá optimizar la toma de decisiones en la gestión de los recursos humanos 
ante esta problemática. Así, atendiendo a los resultados que se desprenden de la investigación desarrollada en este trabajo (resumida en la tabla 5), se puede concluir que el trabajador absentista responde fundamentalmente a una persona de sexo femenino, no cualificada, que se encarga directamente de la fabricación de productos (mano de obra directa o direct labour) realizando tareas estrictamente programadas sujetas a control de métodos y tiempos, que acostumbran a ser rutinarias y repetitivas, y dentro de un proceso de producción complejo (como el descrito para la línea B58 de la empresa base de este trabajo).

Por el contrario, la tendencia absentista parece ser menor en trabajadores varones, y a medida que aumenta su cualificación (dentro de los no cualificados), que llevan a cabo trabajos de apoyo que no suponen una implicación directa en la fabricación de productos, con tareas no programadas de forma estricta dada su naturaleza menos rutinaria y repetitiva, no sujetas a controles habituales de métodos y tiempos. Este menor absentismo parece también ser más propio en trabajadores que ejecutan tareas menos complejas (como las descritas en la línea B9).

Sin embargo, no se ha podido establecer un perfil absentista en base a variables con componente temporal, cuales son la antigüedad en el puesto, la retribución salarial o la edad. Tampoco se han podido extraer conclusiones en cuanto al personal de estructura, quizás por suponer estos casos tan sólo el $16 \%$ de las observaciones realizadas.

La observancia cotidiana y la proximidad a la población estudiada permiten justificar los distintos comportamientos absentistas atendiendo al sexo en base a la falta de flexibilidad horaria, que presenta efectos más nocivos sobre las mujeres que sobre los hombres. En efecto, pese a que en la actualidad las familias parecen alejarse mucho del modelo de familia nuclear parsoniana, donde el hombre se encarga de procurar el sustento económico de la familia y la mujer es quien se ocupa del cuidado y crianza de los hijos, sin embargo, la realidad apunta hacia otro lado, con una distribución todavía desequilibrada del tiempo doméstico, que supone una sobrecarga de tareas para la mujer. Además, este desequilibrio resulta más acusado cuando se combina con bajos niveles de cualificación.

Por este motivo, se derivan de este estudio implicaciones gerenciales que apuntan a una imprescindible e indiscutible implementación de políticas de Responsabilidad Social Corporativa dirigidas hacia los recursos humanos de la empresa, en aras de evitar los problemas derivados del conflicto interrol trabajo-familia. La articulación de estas acciones pasaría por el diseño y puesta en práctica de políticas de conciliación familiar basadas en la flexibilidad laboral y la búsqueda de incentivos que potencien la satisfacción de los recursos humanos, así como prestar una especial atención a las actuaciones en materia de prevención de riesgos laborales (PRL) que vayan más allá de lo establecido en la legislación vigente en la materia. La efectividad de estas medidas tiene repercusiones positivas sobre el compromiso de los empleados con la compañía y la reducción da la intención de abandono.

Las limitaciones atribuibles a esta investigación residen en el ámbito meto- 
dológico. Si bien el recurso metodológico utilizado ha permitido cumplir con los objetivos de la investigación mediante la correlación de variables y la explicación del fenómeno absentista en el caso particular de una planta industrial del sector auxiliar del automóvil sita en España; sin embargo, esta metodología no permite una comprensión del fenómeno absentista, lo cual ha sido suplido mediante la revisión de la literatura desarrollada.

Considerando que los factores sicosociales son la base de una buena prevención para evitar el absentismo motivado por ILT, a raíz de este trabajo se abren futuras líneas que mejoren y completen los resultados aquí alcanzados. Habida cuenta que los problemas de salud más comunicados por los trabajadores están, todos ellos, relacionados en mayor o menor medida con los riesgos sicosociales: dolor de espalda ( $33 \%$ ), estrés ( $28 \%)$, dolores musculares en cuello y hombros $(23 \%)$, y burn-out (23\%); y que existe una clara correlación entre la intensidad del trabajo y los problemas de salud y accidentes, estas nuevas líneas de investigación se fundamentarían en la exploración de número más amplio y diverso de estos factores sicosociales y su evaluación en las empresas donde el índice de absentismo resulte ser superior al del Sector $y$, de este modo, tomar conciencia de las causas que la originan. La necesidad de estas investigaciones resulta palmaria ante los resultados arrojados por la encuesta Europea de Condiciones de Trabajo en 2000 , que muestra que el $57 \%$ de los trabajadores efectúan tareas repetitivas, más de la mitad están sujetos a ritmos de trabajo elevados y con plazos cortos y estrictos durante, al menos, la cuar- ta parte de su jornada laboral, el $21 \%$ del conjunto de trabajadores considera no disponer de tiempo suficiente para realizar su trabajo (Eurofound, 2001). Asimismo, sería deseable que estas investigaciones futuras incorporasen otro tipo de metodología cuantitativa (por ejemplo, ecuaciones estructurales con efecto moderador de las variables de corte sociológico aquí estudiadas) y recurrir, también, a trabajos con base en recursos metodológicos cualitativos, puesto que enriquecerían la comprensión e interpretación del fenómeno absentista.

\section{Referencias bibliográficas}

Arsenault, T. Andre. y Dolan, Shimon (1983). The role of personality, occupation and organization in understanding the relationship between job stress, performance and absenteeism. The Journal of Occupational Psychology. Vol. 56, pp. 227-240.

Allen, Steven (1981). An empirical model of work attendance. Review of Economics and Statistics. Vol. 63, pp. 77-87.

Barmby, Tim A., Orme, Chris D. y Treble, Jhon G. (1991). Worker absenteeism: An analysis using microdata. The Economic Journal. Vol. 101, pp. 214229.

Battisti, Michele y Vallanti, Giovanna (2013). Flexible wage contracts, temporary jobs, and firm performance: Evidence from Italian firms. Industrial Relation. Vol. 52, pp. 737-764.

Bayo, Alberto y Huerta, Emilio (2000). Incentivos y absentismo: un análisis empírico de las empresas industriales españolas. III Encuentro de Economía Aplicada (Valencia-España). En http://goo.gl/nE5eG0 (Consultado el 10 marzo de 2012). 
Bayo Moriones, José Alberto (2003). Los determinantes del absentismo en las empresas industriales españolas. Revista Europea de Dirección y Economía de la Empresa. Vol. 12, pp. 155-172.

Behrend, Héctor (1959). La ausencia voluntaria al trabajo. Revista internacional del trabajo. Vol. 2, pp. 159-170.

Bernardin, H. John (1977). The relationship of personality variables to organizational withdrawal. Personnel Psychology. Vol. 30, pp. 17-27.

Blumenfeld, Dennis.E. y Inman, Robert. R. (2009). Impact of absenteeism on assembly line quality and throughput. Production \& Operations Management. Vol. 18, pp. 333-343.

Boada, Joan, De Diego, Raúl y Agulló, Esteban (2004). El burnout y las manifestaciones psicosomáticas como consecuentes del clima organizacional y de la motivación laboral. Psicothema. Vol. 16, pp. 125-131.

Bourbonnais, Renée. (2005). Psychosocial work environment and certified sick leave among nurses during organizational changes and downsizing. Relations Industrielles. Vol. 60, pp. 483509.

Brooke, Paul P. (1986). Beyond the Steers and Rhodes model of employee attendance. Academy of Management Review. Vol. 11, pp. 345-361.

Brooke, Paul P. y Price, James L. (1989). The determinants of employee absenteeism: An empirical test of a causal model. Journal of Occupational Psychology. Vol. 62, pp. 1-19.

Chadwik-Jones, John K.; Nicholson, Nigel y Brown, Colin A. (1982). Social Psychology of absenteeisim. New York: Praeger

CNAE (Clasificación Nacional de Actividades Económicas) (2009). CNAE 2009. Clasificación Nacional de Activida- des Económicas. En http://goo.gl/ 66TIfn (Consultado el 14 marzo de 2012).

Curry, James P., Wakefield, Douglas S., Price, James L., Mueller, Charles W. y McCloskey, Joanne C. (1985). Determinants of turnover among nursing department personnel. Research in Nursing and Health. Vol.8, pp. 397-411.

Dalton, Dan R. y Mesch, Debra J. (1991). On the extent and reduction of avoidable absenteeism: An assessment of absence policy provisions. Journal of Applied Psychology. Vol. 76, pp. 810-817.

Deaux, Kay y Lewis, Laurie L. (1984). Structure of gender stereotypes: Interrelationships among components and gender label. Journal of Personality and Social Psychology. Vol. 46, pp. 991-1004.

Drago, Robert y Wooden, Mark (1992). The determinants of labor absence: Economic factors and workgroup norms across countries. Industrial and Labor Relations Review. Vol. 45, pp. 764-778.

Dunn, Lucia F. y Youngblood, Stuart A. (1986). Absenteeism as a mechanism for approaching an optimal labour market equilibrium: An empirical study. The Review of Economics and Statistics. Vol. 68, pp. 668-674.

Edwards, Paul K. y Scullion, Hugh (1982). The Social Organisation of Industrial Conflict. Control and Resistance in the Workplace. Oxford: Blackwell.

Engström, Lars Gunnar y Janson, Staffan (2009). Predictors of work presence: Sickness absence in a salutogenic perspective. Work. Vol. 33, pp. 287295

Eurofound (European Foundation for the Improvement of Living and Working Conditions) (2001). Third European survey on working conditions 
2000. En http://www.eurofound.europa.eu/pubdocs/2001/21/en/1/ef0121 en.pdf (Consultado el 14 marzo de 2012)

Frick, Bernd y Malo, Miguel Ángel (2008). Labor market institutions and individual absenteeism in the European Union: The relative importance of sickness benefit systems and employment protection legislation. Industrial Relations. Vol. 47, pp. 505-529.

Greenhaus, Jeffrey H. y Powell, Gary N. (2006). When work and family are allies: A theory of work-family enrichment. Academy of Management Review. Vol. 31, pp. 72-92.

Hackman, J. Richard y Lawler, Edward E. (1971). Employee reactions to job characteristics. Journal of Applied Psychology. Vol. 55, pp. 259-86.

Hochschilld, Arlie (1989). The Second Shift: Working Parents And The Revolution At Home. New York: Viking.

Ichino, Andrea y Moretti, Enrico (2009). Biological gender differences, absenteeism, and the earnings gap. American Economic Journal: Applied Economics. Vol. 1, pp. 183-218.

Isambert-Jamati, Vivianne (1960). Adaptation au travail et niveau de qualification des femmes salaries. Revue francaise de sociologie. Vol. 1, pp. 45-60.

Iverson, Roderick D., Deery, Stephen J. y Erwin, Peter J. (1995). Absenteeism in the health services sector: A causal model and intervention strategies'. En Riedel, P. y Preston, A. (eds) Managing Absenteeism: Analyzing and Preventing Labour Absence. Canberra: Australian Govemment $\mathrm{Pu}$ blishing Service.

Keller, Robert T. (1983). Predicting absenteeism from prior absenteeism, attitudinal factors, and nonattitudinal factors. Journal of Applied Psychology. Vol. 68, pp. 536-540.
Kristensen, Kai; Juhl, Hans Jørn; Eskildsen, Jacob; Nielsen, Jesper; Frederiksen, Nielsen y Bisgaard, Carsten (2006). Determinants of absenteeism in a large Danish bank. International Journal of Human Resource Management. Vol. 17, pp. 1645-1658.

Lips, Hilary M. (1993). Sex and Gender: An introduction. Mountain View, CA: Mayfield.

Løkke, Ann-Kristina, Eskildsen, Jacob K. y Jensen, Troels Wendelboe (2007). Absenteeism in the Nordic countries. Employee Relations. Vol. 29, pp.16-29.

López-Cordón, María Victoria (1998). Familia, sexo y género en la España moderna. Studia histórica. Historia moderna. Vol. 18, pp. 105-134

Markussen, Simen Markussen, Røed, Knut; Røgeberg, Ole J. y Gaure, Simen Gaure (2011). The anatomy of absenteeism. Journal of Health Economics. Vol. 30, pp. 277-292.

Martocchio, Joseph J. (1989). Age-related differences in employee absenteeism: A metaanalysis. Psychology and Aging. Vol. 4, pp. 409-414.

Mason, Claire M. y Griffin, Mark A. (2003). Group absenteeism and positive affective tone: A longitudinal study. Journal of Organizational Behavior. Vol. 24, pp. 667-687.

Matlin, Margaret (1993). The psychology of women. Nueva York: Harcourt Brace Jovanovich.

Moch, Michael K. y Fitzgibbons, Dale E. (1985). The relationship between absenteeism and production efficiency: An empirical assessment. Journal of Occupational Psychology. Vol. 58, pp. 39-47.

Nova, Pilar (1996). El absentismo laboral como indicador de unas deficientes condiciones de trabajo. Cuadernos de Relaciones Laborales. Vol. 9, pp. 231239. 
Ortiz, Yolanda y Samaniego, Carlos (1995). Reflexiones en torno a la evolución del modelo de Steers y Rhodes sobre absentismo laboral. En L. González, A. de la Torre y J. de Elena (Comps.): Psicología del Trabajo y de las Organizaciones, Gestión de Recursos Humanos y Nuevas Tecnologías. Salamanca: Eudema (pp. 237-246).

Paringer, Lynn (1983). Women and Absenteeism: Health or Economics? The American Economic Review. Vol. 73, pp. 123-127.

Price, James L. (1972). Handbook of Organizational Measurement. Lexington, MA: D.C. Heath

Price, James L. y Mueller, Charles W. (1981), Professional Turnover: The Case Of Nurses. New York: SP Medical and Scientific.

Price, James L. y Mueller, Charles W. (1986). Absenteeism and Turnover of Hospital Employees. Greenwich, CT: JAI Press.

Randon, Sophie; Baret, Christophe y Prioul, Christine (2011). La prévention de l'absentéisme du personnel soignant en gériatrie: du savoir académique à l'action managerial. Revue Management et Avenir. Vol. 49, pp. 133-149.

Rhodes, Susan R. y Steers, Richard M. (1990). Managing Employee Absenteeism. USA: Addison-Wesley.

Rockoff, Jonah E. y Herrmann, Mariesa A. (2012). Does menstruation explain gender gaps in work absenteeism? Journal of Human Resources. Vol. 47, pp. 493-508.

Steers, Richard M. y Rhodes, Susan R. (1978). Major influences on employee attendance: A process Model. Journal
Applied Psychology. Vol. 63, pp. 391-407.

Steers, Richard M. y Rhodes, Susan R. (1984). Knowledge and speculation about absenteeism. En P.S, Goodman, I. y Atkins, R.S. (Eds.): Absenteeism: new approaches to understanding, measuring, and managin employee absence. San Francisco: JosseyBass (pp. 229-275).

Tetrick, Lois E. y LaRocco, James M. (1987). Understanding, prediction, and control as moderators of the relationships between perceived stress, satisfaction, and psychological well-being. Journal of Applied Psychology. Vol. 72, pp. 538-543.

Unger, Rhoda Kesler; Crawford, Mary (1992). Women and gender: A feminist psychology. Nueva York: McGraw-Hill.

Velázquez, Susana (2003). Violencias cotidianas, violencia de género: escuchar, comprender, ayudar. Buenos Aires: Paidós.

Vroom, Victor Harold (1964). Work and Motivation. New York: Wiley

White, J. Kenneth y Ruh, Robert A. (1973). Effects of personal values on the relationship between participation and job attitudes. Administrative Science Quarterly. Vol. 18, pp. 506-517.

Wright, Peter, Ferris, Stephen P., Hiller, Janine S. y Kroll, Mark (1995). Competitiveness through management of diversity: effects on stock price valuation. The Academy of Management Journal. Vol. 38, pp. 272-287.

Zick, Cathleen D. y McCullough, Jane L. (1991). Trends in Married Couples' Time Use: Evidence from 1977-78 and 1987-88. Sex Roles. Vol. 24, pp. 459-487. 